\title{
The Hopfield model revisited: Covariance and Quantization
}

\author{
F. Belgiorno ${ }^{1,2}$, S.L. Cacciatori ${ }^{3,4}$, F. Dalla Piazza ${ }^{5}$ \\ 1 Dipartimento di Matematica, Politecnico di Milano, Piazza Leonardo 32, \\ IT-20133 Milano, Italy \\ 2 and INdAM-GNFM \\ 3 Department of Science and High Technology, Università dell'Insubria, Via \\ Valleggio 11, IT-22100 Como, Italy \\ 4 INFN sezione di Milano, via Celoria 16, IT-20133 Milano, Italy \\ 5 Università "La Sapienza", Dipartimento di Matematica, Piazzale A. Moro 2, \\ I-00185, Roma, Italy
}

\begin{abstract}
There are several possible applications of quantum electrodynamics in dielectric media which require a quantum description for the electromagnetic field interacting with matter fields. The associated quantum models can refer to macroscopic electromagnetic fields or, in alternative, to mesoscopic fields (polarization fields) describing an effective interaction between electromagnetic field and matter fields. We adopt the latter approach, and focus on the Hopfield model for the electromagnetic field in a dielectric dispersive medium in a framework in which space-time dependent mesoscopic parameters occur, like susceptibility, matter resonance frequency, and also coupling between electromagnetic field and polarization field. Our most direct goal is to describe in a phenomenological way a space-time varying dielectric perturbation induced by means of the Kerr effect in nonlinear dielectric media. This extension of the model is implemented by means of a Lorentz-invariant Lagrangian which, for constant microscopic parameters, and in the rest frame, coincides with the standard one. Moreover, we deduce a covariant scalar product and provide a canonical quantization scheme which keeps into account the constraints implicit in the model. Examples of viable applications are indicated.
\end{abstract}

PACS numbers: 03.70.+k,03.65.-w,42.50.Ct

Submitted to: Physica Scripta

Keywords: Hopfield model, relativistic covariance, quantum theory. 


\section{Introduction}

A longstanding field of investigation for quantum field theory is represented by pair creation in external fields or by moving boundaries. We limit ourselves to quote a couple of seminal papers which were published at the birth of modern quantum field theory [1, 2. We are mainly interested in photon pair creation associated with variations of the dielectric constant in a dielectric medium, which has been a subject of important investigations, as e.g. a series of papers by Schwinger concerning a possible relation between dynamical Casimir effect and sonoluminescence $[3$. In this paper, instead of quantizing phenomenologically the electromagnetic field in presence of a dielectric medium (see e.g. 4), we delve into a less phenomenological situation in which dielectric properties are modeled as in the Hopfield model: the electromagnetic field interacts with a set of oscillators reproducing sources for dispersive properties of the electromagnetic field in matter [5, 6, 7, 8, We refer to a more general situation where electric susceptibility, resonance frequencies of the electromagnetic field and also the coupling between electromagnetic field and oscillators (the latter ones can be identified with the mesoscopic polarization fields) depend on space-time variables. In this respect, we can refer our approach to other models which generalize the Hopfield model, see [9, 10, 11, but we stress that we don't take into account absorption in our paper. The latter assumption is reasonable as far as emission phenomena we are interested in are not too near the absorption region, and the focus is pair-creation. Any framework including absorption would imply a much more tricky approach (cf. e.g. 9, 10, 11).

In order to corroborate the physical interest of our model and its canonical quantization, we recall that, by means of the Kerr effect, it is possible to induce dielectric perturbations which propagate in the dielectric medium. These perturbations represent inhomogeneities which arise as a consequence of intense laser pulse propagating in a dielectric medium (see e.g. [12). As a result of microscopic interactions, involved in non-linear electrodynamics, a mean motion of a dielectric perturbation with a different refractive index occurs. Instead of attempting immediately a first principle study on this subject, i.e. instead of working out a microscopic model for this case, we begin considering a semi-phenomenological approach, in which we adopt a Lagrangian model which would be 'first principlebased' apart for the appearance in the Lagrangian of a (phenomenological) contribution to the refractive index arising from the Kerr effect. Interesting results can be still deduced from this framework, as well as a well-defined route for quantizing the model. Our aim is to get a model which allows to deal with sufficiently general situations of interest, as e.g. the ones created by means of the Kerr effect: traveling dielectric perturbations moving with different laws of motions should be allowed. For example, a uniformly moving perturbation (which is characteristic of the Kerr effect), but also an accelerating one and even a rotating one, each of which represents a very interesting benchmark for photon pair creation in external fields (or under changing external conditions). For situations covering the uniformly moving case, its relation with analogue gravity framework and for experimental measure of Hawking radiation, see e.g. 13, 14, 15, 16, 17, 18, 19. Thus our approach allows as well the cases where a different space-time evolution is taken into account. As a consequence of microscopic interactions, modified electric susceptibility, proper frequency and coupling constant between electric field and polarization are postulated to occur, which are meant to reproduce a suitable behaviour of the refractive index perturbation.

In the actual construction of the model, we can refer to the aforementioned traveling perturbations, but, in order to be even much more general, we can allow a generic behavior for the susceptibility $\chi(t, x, y, z)$, which is a scalar function of the space-time coordinates. Moreover, we allow an analogous nature also for $\omega_{0}(t, x, y, z)$, the latter being the proper frequency of the matter oscillators. As e.g. in 9, 10, 11, we generalize the coupling between electric field and polarization field(s), in our case by introducing a scalar function $g(t, x, y, z)$ which plays the role of coupling.

The only reasonable constraint we impose are that asymptotically in time $\chi, \omega, g$ are constant, for obtaining well-defined particle states IN and OUT. Moreover, we can allow couplings with $\mathrm{N}$ oscillators, in such a way that we can manage with $\mathrm{N}$ different susceptibilities, resonances and couplings.

There are two further features of our model which are very important: relativistic covariance and quantization in a covariant gauge. Covariance is fundamental for gettting rid of any ambiguity in actual calculations, and in order to respect a fundamental 
requirement for a physical model. We stress that these are per sè interesting contributions to the microscopically-grounded works on the subject of electromagnetic field in dielectric media, because a covariant generalization is presented and its canonical quantization in a covariant gauge is performed. Covariance, as is known, and is confirmed since the original work by Minkowski 20] and e.g. by 21, 22, is not simply a speculative exercise in the picture at hand, but allows to get the correct behavior of physical quantities when changing from a inertial observer to another one. An important example is represented by a uniformly travelling perturbation $v=$ const moving along the $x$-direction, where we can use covariance for passing to the comoving frame, where the theory is static, and where physical interpretation of the scattering process is much more perspicuous 23 .

It is also worth mentioning a longstanding series of studies concerning electrodynamics of moving media, where covariance of the formalism plays a key-role. We limit ourselves to quote some seminal papers 24, 25, 26, 27, where phenomenological electrodynamics is adopted (ref. [27] is explicitly devoted to the dispersive case). In particular, 24] and 26] are also important references for the dielectric models in analogue gravity 28. See also 29, where a covariant model for moving (homogeneous) dispersive dielectric media is studied and quantized in the Coulomb gauge.

As to quantization, we can provide a scheme of constrained quantization where all subtleties of the theory are taken into account (see e.g. [30, 31]), and still covariance plays an important role for obtaining consistent quantization rules which would not be so clearly stated otherwise. As far as dispersive effects are not involving magnetic properties of the material, we can also consider that our model, for constant dielectric susceptibility, represents an improvement of the phenomenological model studied in 32 .

It is worth mentioning that a very general and interesting picture is provided in [11, where the susceptibility is a tensor field depending on space and time. Absorption is also included by means of a bath of oscillators whose interactions with the electromagnetic field originate dissipative effects. Still, as a novelty with respect to the aforementioned picture, we develop a formalism leaving room for covariance and also quantization in a covariant gauge, which are not treated therein.

\section{A covariant form for the Hopfield model}

In the following, we take into consideration the electromagnetic field Lagrangian which is apt for a dispersive lossless dielectric medium, as in Hopfield model 5, 6, 7. A further field, representing material polarization, is introduced and coupled to the free electromagnetic field as follows:

$$
\begin{aligned}
\mathcal{L}_{e m}: & =\frac{1}{8 \pi}\left(\frac{1}{c} \dot{\mathbf{A}}+\nabla \varphi\right)^{2}-\frac{1}{8 \pi}(\nabla \wedge \mathbf{A})^{2} \\
& +\frac{1}{2 \chi \omega_{0}^{2}}\left(\dot{\mathbf{P}}^{2}-\omega_{0}^{2} \mathbf{P}^{2}\right)-\frac{g}{2 c}(\mathbf{P} \cdot \dot{\mathbf{A}}+\dot{\mathbf{A}} \cdot \mathbf{P}) \\
& -\frac{g}{2}(\mathbf{P} \cdot \nabla \varphi+\nabla \varphi \cdot \mathbf{P}) .
\end{aligned}
$$

As an example, a traveling perturbation is described by introducing $\chi(x-v t, y, z), \omega_{0}(x-v t, y, z), g(x-v t, y, z)$. Only a first-principle introduction of the Kerr effect, like e.g. the one obtained by introducing a fourth power of $\mathbf{P}$ would require substantial modifications (because of the non-linear term), but we do not pursue this problem herein.

In order to pursue a more standard calculation for inferring particle creation, we introduce a covariant generalization of the Hopfield model in $3+1$ dimensions: this would make easier to find out an inner product with respect to which one could calculate the Bogoliubov coefficients in order to check if particle creation occurs in the standard way. The problem consists, of course, in finding a covariant form for the polarization part of the Hopfield Lagrangian. This is not a so trivial task. The main problem is represented by the kinetic part of the polarization field Lagrangian; with this aim, let us introduce $v^{\mu}$ as the 4-velocity of the bulk dielectric medium (we mean the velocity of the dielectric sample, not the one of the dielectric perturbation). Then, the covariant lagrangian density is:

$$
\begin{aligned}
\mathcal{L}= & -\frac{1}{16 \pi} F_{\mu \nu} F^{\mu \nu}-\frac{1}{2 \chi \omega_{0}^{2}}\left[\left(v^{\rho} \partial_{\rho} P_{\mu}\right)\left(v^{\sigma} \partial_{\sigma} P^{\mu}\right)\right] \\
& +\frac{1}{2 \chi} P_{\mu} P^{\mu}-\frac{g}{2 c}\left(v_{\mu} P_{\nu}-v_{\nu} P_{\mu}\right) F^{\mu \nu}
\end{aligned}
$$

Minkowski metric $\eta_{\mu \nu}$ is chosen with the standard signature for quantum field theory: $(+,-,-,-)$. The latter model appears to be the principal candidate in our consideration, because it provides a field equation for $P_{\mu}$ which, for constant $\chi$, gives rise e.g. in the eikonal approximation to the correct covariant dispersion relation.

We have introduced a 4-vector in order to describe the polarization field in a covariant form. We have introduced, as a consequence, also the component $P_{0}$ of the field, which is absent in the rest frame. Actually, this new component is not an independent one, it depends on the spatial components, as can be easily ascertained by constructing the 4 -vector corresponding to the standard polarization field, see e.g. [22, 33]. Indeed, the following condition has to be implemented:

$v^{\mu} P_{\mu}=0$,

which is required at the level of the classical theory for the polarization vector. Note that in the rest frame, where $v^{\mu}=(c, 0,0,0)$, the above condition amounts 
to $P_{0}=0$. We assume this condition too. It is worth mentioning that this condition is the correct one for our harmonic oscillator field $P$ coupled to the electromagnetic field, regardless of its specific nature of polarization field.

As to the field equations, in the case of the electromagnetic field we obtain

$$
-\frac{1}{4 \pi} \partial_{\nu} F^{\nu \mu}-v^{\nu} \partial_{\nu}\left(\frac{g}{c} P^{\mu}\right)+v^{\mu} \partial_{\nu}\left(\frac{g}{c} P^{\nu}\right)=0,
$$

and for the polarization field we have

$$
-v^{\alpha} \partial_{\alpha}\left(\frac{1}{\chi \omega_{0}^{2}}\right) v^{\beta} \partial_{\beta} P^{\nu}-\frac{1}{\chi} P^{\nu}+\frac{g}{c} v_{\rho} F^{\rho \nu}=0 .
$$

By contracting with $v_{\mu}$ the equation for the electromagnetic field and taking into account (2) we get $\partial_{\mu}\left(E^{\mu}+4 \pi \frac{g}{c} P^{\mu}\right)=0$, where $E^{\mu}:=v_{\nu} F^{\nu \mu}$. This is just the Gauss law for the electric induction field $D^{\mu}:=E^{\mu}+4 \pi \frac{g}{c} P^{\mu}$. This is the right condition to identify $P^{\mu}$ as a polarization field and is required by compatibility among the transversality condition and the equations of motion. It is also useful to define the induction tensor $G^{\mu \nu}$, which is such that $D^{\nu}=v_{\mu} G^{\mu \nu}, H_{\mu}=\frac{1}{2} v^{\nu} \epsilon_{\mu \nu \rho \sigma} G^{\rho \sigma}:=\frac{1}{2} v^{\nu} \epsilon_{\mu \nu \rho \sigma} F^{\rho \sigma}=$ $B_{\mu}$. Notice that, in absence of free charges and currents, Gauss law and Ampere law are summarized by the equation $\partial_{\mu} G^{\mu \nu}=0$, which, contracted with $v_{\nu}$, amounts to $\partial_{\mu}\left(E^{\mu}+4 \pi \frac{g}{c} P^{\mu}\right)=0$.

It is also easy to realize that, by introducing the $\Phi_{\mu}:=\left(A_{\mu}, P_{\mu}\right)$, whose first four components coincide with $A_{\mu}$ and the remaining four components coincide with $P_{\mu}$ 甘 we obtain a field theory which is quadratic in $\Phi_{\mu}$.

\section{Conserved scalar product for the model}

We can now determine the conserved scalar product associated to the covariant Hopfield model. The first step in order to determine the scalar product is to complexify the fields. The complexified lagrangian density becomes:

$$
\begin{aligned}
\mathcal{L}_{e m}^{c o v} & =-\frac{1}{16 \pi} F_{\mu \nu}^{*} F^{\mu \nu}-\frac{1}{2 \chi \omega_{0}^{2}}\left[\left(v^{\rho} \partial_{\rho} P_{\mu}^{*}\right)\left(v^{\sigma} \partial_{\sigma} P^{\mu}\right)\right. \\
& \left.-\omega_{0}^{2} P_{\mu}^{*} P^{\mu}\right]-\frac{g}{2 c} P_{\mu}^{*} v_{\rho} F^{\rho \mu}-\frac{g}{2 c} P^{\mu} v^{\rho} F_{\rho \mu}^{*} .
\end{aligned}
$$

A symmetry of (5) is $A_{\mu} \mapsto e^{i \phi} A_{\mu}, P_{\mu} \mapsto e^{i \phi} P_{\mu}, A_{\mu}^{*} \mapsto$ $e^{-i \phi} A_{\mu}^{*}, P_{\mu}^{*} \mapsto e^{-i \phi} P_{\mu}^{*}$, where $\phi$ is a constant phase. The associated conserved quantity can be computed by means of the usual Noether method. The computation is immediate and gives the conserved current:

$$
\begin{aligned}
\mathcal{J}^{\mu} & =\frac{i}{2}\left[\frac{1}{4 \pi} F^{* \mu \nu} A_{\nu}+\frac{1}{\chi \omega_{0}^{2}} v^{\rho} \partial_{\rho} P^{* \sigma} P_{\sigma} v^{\mu}\right. \\
& \left.-\frac{g}{c}\left(P^{* \mu} v^{\rho}-P^{* \rho} v^{\mu}\right) A_{\rho}-c . c .\right] .
\end{aligned}
$$

$\ddagger \Phi_{\mu}$ is the direct sum of $A_{\mu}$ and $P_{\mu}$.
Indeed, a direct computation shows that on the solutions of the equations of motion $\mathcal{J}^{\mu}$ satisfies $\partial_{\mu} \mathcal{J}^{\mu}=0$. Thus, the standard argument shows that on any spacelike slice $\Sigma_{t}$ the quantity $Q:=\int_{\Sigma_{t}} \mathcal{J}^{0} d^{3} x$ does not depend from $t$. This defines a conserved (Hermitian) quadratic form $Q$ on $\Phi_{\mu}=\left(A_{\mu}, P_{\mu}\right)$ :

$$
\begin{aligned}
Q\left(\left(A_{\mu}, P_{\mu}\right)\right) & =\frac{i}{2} \int_{\Sigma_{t}}\left[\frac{1}{4 \pi} F^{* 0 \nu} A_{\nu}+\frac{1}{\chi \omega_{0}^{2}} v^{\rho} \partial_{\rho} P^{* \sigma} P_{\sigma} v^{0}\right. \\
& \left.-\frac{g}{c}\left(P^{* 0} v^{\rho}-P^{* \rho} v^{0}\right) A_{\rho}-\text { c.c. }\right] d^{3} x .
\end{aligned}
$$

This gives the conserved scalar product by means of the usual polarization formula. Then we obtain the conserved scalar product:

$$
\begin{aligned}
& \left(\left(A_{\mu}, P_{\mu}\right) \mid\left(\tilde{A}_{\mu}, \tilde{P}_{\mu}\right)\right) \\
& =\frac{i}{2} \int_{\Sigma_{t}}\left[\frac{1}{4 \pi} F^{* 0 \nu} \tilde{A}_{\nu}+\frac{1}{\chi \omega_{0}^{2}} v^{\rho} \partial_{\rho} P^{* \sigma} \tilde{P}_{\sigma} v^{0}\right. \\
& -\frac{g}{c}\left(P^{* 0} v^{\rho}-P^{* \rho} v^{0}\right) \tilde{A}_{\rho}-\frac{1}{4 \pi} \tilde{F}^{0 \nu} A_{\nu}^{*} \\
& \left.-\frac{1}{\chi \omega_{0}^{2}} v^{\rho} \partial_{\rho} \tilde{P}^{\sigma} P_{\sigma}^{*} v^{0}+\frac{g}{c}\left(\tilde{P}^{0} v^{\rho}-\tilde{P}^{\rho} v^{0}\right) A_{\rho}^{*}\right] d^{3} x .
\end{aligned}
$$

This scalar product is very important in relation to the quantization of the model. Indeed, it allows to define positive and negative norm states for the solutions of the field equations, i.e. it allows to define in a proper way particles and antiparticles respectively. A proper quantization for the model is discussed in the following section.

For example, if $\chi(t, \mathbf{x})=\chi_{0}, \omega_{0}(t, \mathbf{x})=\omega_{0}, g(t, \mathbf{x})=1$ and we work in the lab frame, the scalar product among plane waves (with on shell momenta)

$$
\begin{aligned}
\left(A^{\mu}, P^{\mu}\right) & =\left(\mathcal{A}^{\mu} e^{-i \omega t+i \mathbf{k} \cdot \mathbf{x}},\right. \\
& \left.-i \frac{\chi_{0}}{\omega_{0}^{2}-\omega^{2}} \frac{\omega}{c} \mathcal{A}^{\mu} e^{-i \omega t+i \mathbf{k} \cdot \mathbf{x}}\right),
\end{aligned}
$$

and

$$
\begin{aligned}
\left(\tilde{A}^{\mu}, \tilde{P}^{\mu}\right) & =\left(\tilde{\mathcal{A}}^{\mu} e^{-i \tilde{\omega} t+i \tilde{\mathbf{k}} \cdot \mathbf{x}},\right. \\
& \left.-i \frac{\chi_{0}}{\omega_{0}^{2}-\tilde{\omega}^{2}} \frac{\tilde{\omega}}{c} \tilde{\mathcal{A}}^{\mu} e^{-i \tilde{\omega} t+i \tilde{\mathbf{k}} \cdot \mathbf{x}}\right),
\end{aligned}
$$

where $\mathcal{A}^{\mu}, \tilde{\mathcal{A}}^{\mu}$ in (9) and in (10) stand for constant 4 -vectors representing the amplitude of the respective plane waves, is

$$
\begin{aligned}
& \left(\left(A_{\mu}, P_{\mu}\right) \mid\left(\tilde{A}_{\mu}, \tilde{P}_{\mu}\right)\right) \\
= & \frac{\omega}{c}\left[\frac{1}{4 \pi}+\frac{\chi_{0} \omega_{0}^{2}}{\left(\omega_{0}^{2}-\omega^{2}\right)^{2}}\right] \mathbf{A}^{*} \cdot \tilde{\mathbf{A}} \delta^{(3)}(\mathbf{k}-\tilde{\mathbf{k}}),
\end{aligned}
$$

where $A^{\mu}=(0, \mathbf{A})$ and $\tilde{A}^{\mu}=(0, \tilde{\mathbf{A}})$.

\section{Quantization of the covariant model}

The introduction of the condition (2) amounts to a constraint to be imposed on the system. This affects also the quantization of the covariant Hopfield model, in the sense that the covariant form of the Heisenberg commutation relations has to be consistent with the 
constraints of the theory.

As to the electromagnetic part of the Lagrangian, the procedure we follow slightly departs from what could be considered as standard, e.g. the usual quantization under covariant gauge conditions like the Lorentz gauge $\partial_{\mu} A^{\mu}=0$, and where a Gupta-Bleuler formalism (see e.g. 34]) can be adopted in order to get rid of spurious degrees of freedom, leaving only transverse (physical) ones. Instead, we follow the Dirac approach in which all first-class constraints which are associated with the gauge freedom are first reduced to second-class ones by means of suitable explicit gaugefixing terms in the Lagrange formalism, and then quantized [35, 36]. Constraints are then implemented operatorially, rather than in a weak sense (as in the Gupta-Bleuler approach). See e.g. 30, 31, 37, 38.

In order to perform a complete quantization of the full Hopfield covariant model, in a covariant gauge, we have to take into account both the electromagnetic part and the polarization part of the Lagrangian. Both these parts require a suitable implementation of the constraints. We can add the constraints to the covariant Lagrangian, thus obtaining:

$$
\begin{aligned}
\mathcal{L}_{c}:= & -\frac{1}{16 \pi} F_{\mu \nu} F^{\mu \nu}-\frac{1}{2 \chi \omega_{0}^{2}}\left[\left(v^{\rho} \partial_{\rho} P_{\mu}\right)\left(v^{\sigma} \partial_{\sigma} P^{\mu}\right)\right] \\
& +\frac{1}{2 \chi} P_{\mu} P^{\mu}-\frac{g}{2 c}\left(v_{\mu} P_{\nu}-v_{\nu} P_{\mu}\right) F^{\mu \nu} \\
& +B\left(\partial_{\mu} A^{\mu}\right)+\frac{\xi}{2} B^{2}+\lambda\left(v_{\mu} P^{\mu}\right),
\end{aligned}
$$

where $B$ plays the usual role of auxiliary hermitian scalar field, also known as $B$-field [41, 30, and $\xi$ is a constant which is useful for reproducing various gauge conditions (the so-called $R_{\xi}$-gauges). The equations of motions are

$$
\begin{aligned}
& -\frac{1}{4 \pi} \partial_{\nu} F^{\nu \mu}-v^{\nu} \partial_{\nu}\left(\frac{g}{c} P^{\mu}\right)+v^{\mu} \partial_{\nu}\left(\frac{g}{c} P^{\nu}\right) \\
& +\partial^{\mu} B=0, \\
& -v^{\alpha} \partial_{\alpha}\left(\frac{1}{\chi \omega_{0}^{2}}\right) v^{\beta} \partial_{\beta} P^{\nu}-\frac{1}{\chi} P^{\nu}+\frac{g}{c} v_{\rho} F^{\rho \nu} \\
& +\lambda v^{\mu}=0, \\
& \partial_{\nu} A^{\nu}+\xi B=0 .
\end{aligned}
$$

Furthermore, as far as the conjugate momenta are concerned, we obtain:

$$
\begin{aligned}
& \frac{\partial \mathcal{L}_{c}}{\partial \partial_{t} A_{0}}=: \Pi_{A}^{0}= \frac{B}{c}, \\
& \frac{\partial \mathcal{L}_{c}}{\partial \partial_{t} A_{i}}=: \Pi_{A}^{i}=-\frac{1}{4 \pi c}\left(\partial^{0} A^{i}-\partial^{i} A^{0}\right) \\
& \quad-\frac{g}{c^{2}}\left(v^{0} P^{i}-v^{i} P^{0}\right), \\
& \frac{\partial \mathcal{L}_{c}}{\partial \partial_{t} B}=: \pi_{B}=0, \\
& \frac{\partial \mathcal{L}_{c}}{\partial \partial_{t} P_{\mu}}=: \Pi_{P}^{\mu}=-\frac{1}{\chi \omega_{0}^{2} c} v^{0} v^{\sigma} \partial_{\sigma} P^{\mu}, \\
& \frac{\partial \mathcal{L}_{c}}{\partial \partial_{t} \lambda}=: \pi_{\lambda}=0 .
\end{aligned}
$$

As to the classical Hamiltonian density, according to the standard procedure we have:

$$
\begin{aligned}
\mathcal{H} & =\left(\partial_{t} A^{\mu}\right) \Pi_{A \mu}+\left(\partial_{t} P^{\mu}\right) \Pi_{P \mu}-\mathcal{L}_{c}+u \pi_{\lambda} \\
& +y \pi_{B}+z\left(\Pi_{A}^{0}-\frac{B}{c}\right) \\
& =2 \pi c^{2}\left(\Pi_{A}^{i}\right)^{2}+\frac{1}{16 \pi} F_{i j} F^{i j}+c A_{0}\left(\partial_{i} \Pi_{A i}\right) \\
& +4 \pi g\left(v_{0} P_{i}-v_{i} P_{0}\right) \Pi_{A i}-c \frac{v^{k}}{v_{0}}\left(\partial_{k} P^{\mu}\right) \Pi_{P \mu} \\
& -\frac{\chi \omega_{0}^{2} c^{2}}{2\left(v^{0}\right)^{2}} \Pi_{P} \Pi_{P}^{\mu}-\frac{1}{2 \chi} P_{\mu} P^{\mu}+\frac{2 \pi g^{2}}{c^{2}}\left(v_{0} P_{i}-v_{i} P_{0}\right)^{2} \\
& +\frac{g}{2 c}\left(v_{i} P_{j}-v_{j} P_{i}\right) F^{i j}-B\left(\partial_{i} A^{i}\right)-\frac{\xi}{2} B^{2} \\
& -\lambda\left(v_{\mu} P^{\mu}\right)+u \pi_{\lambda}+y \pi_{B}+z\left(\Pi_{A}^{0}-\frac{B}{c}\right) .
\end{aligned}
$$

In our model there are three primary constraints $\pi_{B}, \Pi_{A}^{0}-B / c, \pi_{\lambda}$. From the analysis of Poisson brackets of the primary constraints with the Hamiltonian we can find the following complete set of constraints:

$\Gamma_{1}=\pi_{B}$,

$\Gamma_{2}=\Pi_{A}^{0}-\frac{B}{c}$,

$\Gamma_{3}=v_{\mu} P^{\mu}$,

$\Gamma_{4}=v_{\mu} \Pi_{P}^{\mu}$,

$\Gamma_{5}=\lambda$,

$\Gamma_{6}=\pi_{\lambda}$.

We have taken into account that all functions of the constraints giving rise to the same submanifold $\Gamma_{i}=0, i=1,2,3,4,5,6$ are to be considered equivalent, and this allows us to get the former simplified expressions for $\Gamma_{4}, \Gamma_{5}$. See also [31, 30. $\Gamma_{1}, \Gamma_{2}, \Gamma_{6}$ represent primary second-class constraints of the theory, and they appear explicitly in the expression of our constrained Hamiltonian defined above. We provide in the following some more details of the calculations.

In order to be more explicit, we get

$$
\begin{aligned}
\left\{\pi_{B}, H\right\} & =\partial_{i} A^{i}+\xi B+\frac{z}{c}, \\
\left\{\Pi_{A}^{0}-B, H\right\} & =-c \partial_{i} \Pi_{A i}-y, \\
\left\{\pi_{\lambda}, H\right\} & =-v_{\mu} P^{\mu},
\end{aligned}
$$

which represent the Poisson brackets with the primary second-class constraints. The first equation (28) fixes $z$ and is the same one obtains in QED [30, and implies the same conditions. The second equation (29) determines $y$. The third equation (30) introduces a second-class second-stage constraint. Its Poisson bracket with the Hamiltonian is

$\left\{v_{\mu} P^{\mu}, H\right\}=-\frac{\chi \omega_{0}^{2} c^{2}}{\left(v^{0}\right)^{2}} v_{\mu} \Pi_{P}^{\mu}-c \frac{v^{k}}{v^{0}} \partial_{k}\left(v_{\mu} P^{\mu}\right)$

which, on the sub manifold defined by the above constraints amounts to requiring $v_{\mu} \Pi_{P}^{\mu}=0$, which is 
then a second-class third-step constraint. Its Poisson bracket with the Hamiltonian is

$\left\{v_{\mu} \Pi_{P}^{\mu}, H\right\}=\frac{1}{\chi} v_{\mu} P^{\mu}+c \frac{v^{k}}{v^{0}}\left(\partial_{k} \delta\right)\left(v_{\mu} \Pi_{P}^{\mu}\right)+v^{\mu} v_{\mu} \lambda$,

which, on the submanifold of the previous constraints implies $\lambda=0$. At this point we have a complete set of constraints, because

$\{\lambda, H\}=u$

simply fixes $u=0$. See also below.

We can define the matrix $\left\{C_{i j}\right\}$ whose entries are $C_{i j}:=\left\{\Gamma_{i}, \Gamma_{j}\right\}$, where $\{$,$\} stay for the Poisson brack-$ ets. In particular, restoring the dependence on spacetime variables (time is fixed), with $x=(t, \mathbf{x})$, we get the following non-zero entries: $\left\{\Gamma_{1}(t, \mathbf{x}), \Gamma_{2}(t, \mathbf{y})\right\}=$ $\delta^{(3)}(\mathbf{x}-\mathbf{y}) / c, \quad\left\{\Gamma_{3}(t, \mathbf{x}), \Gamma_{4}(t, \mathbf{y})\right\}=v_{\mu} v^{\mu} \delta^{(3)}(\mathbf{x}-$ $\mathbf{y}),\left\{\Gamma_{5}(t, \mathbf{x}), \Gamma_{6}(t, \mathbf{y})\right\}=\delta^{(3)}(\mathbf{x}-\mathbf{y})$. Let us explicit the Dirac brackets as provided from the theory of constrained systems:

$$
\{\mathcal{A}, \mathcal{B}\}_{D}=\{\mathcal{A}, \mathcal{B}\}-\left\{\mathcal{A}, \Gamma_{i}\right\} C_{i j}^{-1}\left\{\Gamma_{j}, \mathcal{B}\right\} .
$$

We recall that, in a less synthetic form, in the previous formula one has to take into account that, by introducing collective symbols for phasespace variables $\left\{X_{l}\right\}=\left\{A^{\mu}, P^{\mu}, B, \lambda\right\}, \quad\left\{\bar{\Pi}_{l}\right\}=$ $\left\{\Pi_{A}^{\mu}, \Pi_{P}^{\mu}, \pi_{B}, \pi_{\lambda}\right\}$, we get:

$$
\begin{aligned}
& \{\mathcal{A}, \mathcal{B}\}=\int d^{3} z\left(\frac{\delta \mathcal{A}}{\delta X_{l}(t, \mathbf{z})} \frac{\delta \mathcal{B}}{\delta \bar{\Pi}_{l}(t, \mathbf{z})}\right. \\
- & \left.\frac{\delta \mathcal{A}}{\delta \bar{\Pi}_{l}(t, \mathbf{z})} \frac{\delta \mathcal{B}}{\delta X_{l}(t, \mathbf{z})}\right) \\
& \left\{\mathcal{A}, \Gamma_{i}\right\} C_{i j}^{-1}\left\{\Gamma_{j}, \mathcal{B}\right\} \\
= & \int d^{3} u d^{3} w\left\{\mathcal{A}, \Gamma_{i}(t, \mathbf{u})\right\} C_{i j}^{-1}(\mathbf{u}, \mathbf{w})\left\{\Gamma_{j}(t, \mathbf{w}), \mathcal{B}\right\} ;
\end{aligned}
$$

a summation convention on repeated indices is understood. One can also determine the Lagrange multipliers $u, y, z$ that appear for primary second-class constraints $\Gamma_{1}, \Gamma_{2}, \Gamma_{6}$ in the constrained Hamiltonian, as shown in our previous discussion. It is now easy to show that the following Dirac brackets hold true:

$$
\begin{aligned}
& \left\{A^{\mu}(t, \mathbf{x}), \Pi_{A}^{\nu}(t, \mathbf{y})\right\}_{D}:=\eta^{\mu \nu} \delta^{(3)}(\mathbf{x}-\mathbf{y}) \\
& \left\{P^{\mu}(t, \mathbf{x}), \Pi_{P}^{\nu}(t, \mathbf{y})\right\}_{D} \\
& :=\left(\eta^{\mu \nu}-\frac{1}{v_{\rho} v^{\rho}} v^{\mu} v^{\nu}\right) \delta^{(3)}(\mathbf{x}-\mathbf{y}) \\
& \left\{B(t, \mathbf{x}), \pi_{B}(t, \mathbf{y})\right\}_{D}:=0 \\
& \left\{\lambda(t, \mathbf{x}), \pi_{\lambda}(t, \mathbf{y})\right\}_{D}:=0
\end{aligned}
$$

which represent the basic ingredients for the quantization. Indeed, according to Dirac quantization scheme, we have to impose on quantum operators:

$$
\left[\hat{X}^{l}(t, \mathbf{x}), \hat{\bar{\Pi}}_{k}(t, \mathbf{y})\right]:=i \hbar\left\{X^{l}(t, \mathbf{x}), \bar{\Pi}_{k}(t, \mathbf{y})\right\}_{D} .
$$

Moreover, constraints $\Gamma_{i}=0, i=1, \ldots, 6$, are implemented as operators (cf. 30], in particular p. 132):

$$
\begin{aligned}
& \hat{\pi}_{B}=0, \\
& \hat{\Pi}_{A}^{0}=\frac{\hat{B}}{c}, \\
& v_{\mu} \hat{P}^{\mu}=0, \\
& v_{\mu} \hat{\Pi}_{P}^{\mu}=0, \\
& \hat{\lambda}=0, \\
& \hat{\pi}_{\lambda}=0 .
\end{aligned}
$$

As to the Hamiltonian, we have

$$
\begin{aligned}
H & =\int d^{3} x\left[2 \pi c^{2}\left(\Pi_{A}^{i}\right)^{2}+\frac{1}{16 \pi} F_{i j} F^{i j}+c A_{0}\left(\partial_{i} \Pi_{A} i\right)\right. \\
& +4 \pi g\left(v_{0} P_{i}-v_{i} P_{0}\right) \Pi_{A}-c \frac{v^{k}}{v_{0}}\left(\partial_{k} P^{\mu}\right) \Pi_{P \mu} \\
& -\frac{\chi \omega_{0}^{2} c^{2}}{2\left(v^{0}\right)^{2}} \Pi_{P \mu} \Pi_{P}^{\mu}-\frac{1}{2 \chi} P_{\mu} P^{\mu}+\frac{2 \pi g^{2}}{c^{2}}\left(v_{0} P_{i}-v_{i} P_{0}\right)^{2} \\
& +\frac{g}{2 c}\left(v_{i} P_{j}-v_{j} P_{i}\right) F^{i j}-B\left(\partial_{i} A^{i}\right)-\frac{\xi}{2} B^{2} \\
& \left.-\lambda\left(v_{\mu} P^{\mu}\right)+u \pi_{\lambda}+y \pi_{B}+z\left(\Pi_{A}^{0}-\frac{\vec{B}}{c}\right)\right]
\end{aligned}
$$

As we are dealing with a model in which only second class primary constraints appear, we can also explicit the Lagrange multipliers as functions of the canonical variables. By taking into account our previous analysis, we can limit our attention only to $y, z$, which display the only non-trivial behaviour, and replace $y=-c \partial_{i} \Pi_{A i}$ (cf. (29)), $z=-c\left(\partial_{i} A^{i}+\xi B\right)($ cf. (28) $)$ in (47). (We recall that $u=0=\lambda$ ).

It is interesting to note that, according to (45), (46), $\lambda$ and its conjugate variable $\pi_{\lambda}$ are 'expelled' by constrained quantization, in the sense that they are reduced both to the zero operator. We can provide the following interpretation. Condition (2) holds true as a consequence of the antisymmetric character of the induction tensor $G^{\mu \nu}$ defined at the end of sec. 2. Indeed, we easily get $v_{\nu} D^{\nu}=v_{\nu} v_{\mu} G^{\mu \nu}=0$, which, being $D^{\mu}:=E^{\mu}+4 \pi \frac{g}{c} P^{\mu}$, and being $v_{\mu} E^{\mu}:=$ $v_{\mu} v_{\nu} F^{\nu \mu}=0$, necessarily implies also $v_{\mu} P^{\mu}=0$. This condition is then preserved by quantization. This fact corroborates the previous results concerning the quantization of the model.

\subsection{Scalar product and constraints}

We notice that we have introduced in our setting constraints even in the Lagrangian approach. As a consequence, we have to take into account how the scalar product (8) is modified because of the constraints. After complexification of the constrained Lagrangian $\mathcal{L}_{c}$, we find that it appears only a further term in the scalar product: if $\left(A_{\mu}, P_{\mu}, B, \lambda\right)$ stays for the direct sum of $A_{\mu}, P_{\mu}, B, \lambda$, we get $\left(\left(A_{\mu}, P_{\mu}, B, \lambda\right) \mid\left(\tilde{A}_{\mu}, \tilde{P}_{\mu}, \tilde{B}, \tilde{\lambda}\right)\right)$ 


$$
\begin{aligned}
& =\frac{i}{2} \int_{\Sigma_{t}}\left[\frac{1}{4 \pi} F^{* 0 \nu} \tilde{A}_{\nu}+\frac{1}{\chi \omega_{0}^{2}} v^{\rho} \partial_{\rho} P^{* \sigma} \tilde{P}_{\sigma} v^{0}\right. \\
& -\frac{g}{c}\left(P^{* 0} v^{\rho}-P^{* \rho} v^{0}\right) \tilde{A}_{\rho}-\frac{1}{4 \pi} \tilde{F}^{0 \nu} A_{\nu}^{*} \\
& -\frac{1}{\chi \omega_{0}^{2}} v^{\rho} \partial_{\rho} \tilde{P}^{\sigma} P_{\sigma}^{*} v^{0}+\frac{g}{c}\left(\tilde{P}^{0} v^{\rho}-\tilde{P}^{\rho} v^{0}\right) A_{\rho}^{*} \\
& \left.-\left(B^{*} \tilde{A}^{0}-\tilde{B} A^{0^{*}}\right)\right] d^{3} x .
\end{aligned}
$$

Notice that, assuming the same fields as in (11), we still obtain the same result.

It is also important to point out that the former scalar product can be found as follows. We introduce the phase-space vectors $\left(\left\{X^{l}\right\},\left\{\bar{\Pi}_{l}\right\}\right)$, to be intended again as a direct sum, by using the symplectic form:

$\Omega:=i\left[\begin{array}{cc}0 & 1_{10 \times 10} \\ -1_{10 \times 10} & 0\end{array}\right]$,

where $1_{10 \times 10}$ stays for the identity matrix $10 \times 10$. Then we can define the following scalar product:

$$
\begin{aligned}
& \left\langle\left(\left\{X^{l}\right\},\left\{\bar{\Pi}_{l}\right\}\right),\left(\left\{\tilde{X}^{l}\right\},\left\{\tilde{\bar{\Pi}}_{l}\right\}\right)\right\rangle \\
& :=\int\left(\left\{X^{* l}, \bar{\Pi}_{l}^{*}\right\}\right) \cdot \Omega\left(\left\{\tilde{X}^{l}\right\},\left\{\tilde{\bar{\Pi}}_{l}\right\}\right) d^{3} x,
\end{aligned}
$$

where $\cdot$ stays for the usual Euclidean scalar product. Then, by taking into account the definitions for $\bar{\Pi}_{l}$ one gets the same result as in (48). This is the extension to a canonical quantization scheme and to $3+1$ dimensions of the results obtained for $1+1$ dimensions in [18.

It is also possible to introduce the operator $\tilde{H}$ such that the Hamiltonian in (47) can be written as follows (see e.g. 39]):

$$
\begin{aligned}
H & =\frac{1}{2}\left(\left(\left\{X^{l}\right\},\left\{\bar{\Pi}_{l}\right\}\right), \tilde{H}\left(\left\{X^{l}\right\},\left\{\bar{\Pi}_{l}\right\}\right)\right) \\
& =\frac{1}{2} \int\left(\left\{X^{* l}, \bar{\Pi}_{l}^{*}\right\}\right) \cdot \tilde{H}\left(\left\{\tilde{X}^{l}\right\},\left\{\tilde{\bar{\Pi}}_{l}\right\}\right) d^{3} x
\end{aligned}
$$

which is such that, by defining $\Psi:=\left(\left\{X^{l}\right\},\left\{\bar{\Pi}_{l}\right\}\right)$, one obtains the Hamiltonian equations in the form

$\dot{\Psi}=-i \Omega\left(\nabla_{\Psi} H\right)=-i \Omega \tilde{H} \Psi$,

where $\nabla_{\Psi}=\left(\left\{\partial_{X^{l}}\right\},\left\{\partial_{\bar{\Pi}_{l}}\right\}\right)$ It is also evident that, due to the trivialization of $\lambda$ and $\pi_{\lambda}$, we can restrict our considerations to a $9 \times 9$ dimensional phase space in a straightforward way.

\subsection{Construction of physical states and physical operators}

In this subsection, we delve into the problem of constructing physical states and observables in our canonical approach in covariant gauges. As wellknown, the price to be paid for covariance is the appearance of negative norm states, and also of zeronorm ones. As in QED, one has to face with the problem of non-physical states, which have to be eliminated from the physical spectrum. Also the Fano diagonalization procedure has to be revisited in view of the particular gauge conditions. In the standard approach to quantum electrodynamics, the calculation of the S-matrix requires several ingredients. Lacking a clear and well-defined way to implement a full non-perturbative quantum field theory in the Heisenberg representation, a perturbative approach is implemented in the interaction representation, where the fields evolve as free fields (i.e. their evolution is prescribed by the free field Hamiltonian $H_{0}$ ). A delicate interplay of the interacting (renormalizable) theory with asymptotic free fields occurs (cf. e.g. 30 for the case of QED). Asymptotic free fields (IN and OUT fields) satisfy canonical commutation relations and their creation and annichilation operators satisfy free-field commutation rules. In the present case, in order to simplify a bit our picture, we limit ourselves to the homogeneous dielectric medium, without losses and without dielectric perturbations e.g. induced by means of the Kerr effect. This is not a so crude limitation, as in region asymptotically far from the traveling dielectric perturbation, homogeneity can be assumed to be preserved. Within the given approximation, we assume a quantization strategy which goes parallel to the one of [40, i.e. we quantize the fields as free, with the coupling $g=0$, and then perform a Fano diagonalization for the physical part of the Hamiltonian (with $g \neq 0$ ). We expect that the procedure is consistent with the original commutation relations for the fields, as is in [40] (cf. also 9]).

Furthermore, we wish to avoid any problem with the dipole ghost (see e.g. [41]) so we impose the Feynman gauge $\xi=4 \pi$ in what follows. See also the following section.

We start from the polarization field. It is a vector field with four components, so a priori it would involve four degrees of freedom. Still, the transversality condition $v^{\mu} P_{\mu}=0$ (2) reduces to three the degrees of freedom which are actually available. This is also evident from (37), which in the rest frame reduces identically to zero. Indeed, the set of eight fields $\left\{P_{\mu}, \partial_{0} P_{\mu}\right\}$ represents a complete but not independent set of initial conditions, because (2) allows to express $P_{0}, \partial_{0} P_{0}$ as a function of the set $\left\{P_{i}, \partial_{0} P_{i}\right\}$ which is both complete and independent. We can choose, in particular, a set of polarization vectors $e_{\lambda}^{\mu}, \lambda=1,2,3$, such that

$$
\sum_{\lambda=1}^{3} e_{\lambda}^{\mu} e_{\lambda}^{\nu}=\eta^{\mu \nu}-\frac{v^{\mu} v^{\nu}}{v_{\rho} v^{\rho}}
$$

which can be easily implemented. See below. As to $P^{\mu}$, we have

$P^{\mu}=\sum_{\lambda=1}^{3} \int d^{4} p \frac{1}{N_{p}} \delta(D R)\left[e_{\lambda}^{\mu} b_{\lambda}(\mathbf{p}) e^{i p x}+\right.$ h.c. $]$,

where $D R$ indicates the free field dispersion relation, and $N_{p}$ is a suitable normalization factor. Our choice 
for the basis is $\left\{e_{1}^{\mu}, e_{2}^{\mu}, \frac{v^{\mu}}{c}-p^{\mu} \frac{c}{\omega}\right\}$, where $\omega=v^{\mu} p_{\mu}$ and

$e_{\lambda}^{\mu}=\left(0, \mathbf{e}_{\lambda}\right), \quad \lambda=1,2$,

in such a way that, for $\lambda=1,2$ it holds $\mathbf{e}_{\lambda} \cdot \mathbf{e}_{\lambda}^{\prime}=\delta_{\lambda \lambda^{\prime}}$, and

$v_{\mu} e_{\lambda}^{\mu}=0$,

$p_{\mu} e_{\lambda}^{\mu}=0$,

where $p^{\mu}$ is the wave-vector, as usual. The third polarization is orthogonal to $e_{1}^{\mu}, e_{2}^{\mu}$, and also to $v^{\mu}$. For the operators $b_{\lambda}(\mathbf{p})$ the following canonical commutation relations (CCR) hold:

$$
\left[b_{\lambda}(\mathbf{p}), b_{\lambda^{\prime}}^{\dagger}(\mathbf{q})\right]=\delta_{\lambda \lambda^{\prime}} \delta^{(3)}(\mathbf{p}-\mathbf{q}) \text {. }
$$

For the electromagnetic field and the auxiliary field $B$ quantization requires some more efforts. We can introduce a further set of polarization vectors $\bar{e}_{\lambda}^{\mu}, \lambda=0,1,2,3$ and of operators $a_{\lambda}(\mathbf{p})$ such that

$A^{\mu}=\sum_{\lambda=0}^{3} \int d^{4} p \frac{1}{W_{p}} \delta(D R)\left[\bar{e}_{\lambda}^{\mu} a_{\lambda}(\mathbf{p}) e^{i p x}+\right.$ h.c. $]$,

where $W_{p}$ is a suitable normalization. Usually, one imposes

$$
\sum_{\lambda=0}^{3} \bar{e}_{\lambda}^{\mu} \bar{e}_{\lambda}^{\nu}=\eta^{\mu \nu} .
$$

In particular, one may choose the set of polarization vectors $\left\{\frac{v^{\mu}}{c}, e_{1}^{\mu}, e_{2}^{\mu}, e_{3}^{\mu}\right\}$, where $e_{\lambda}^{\mu}, \lambda=1,2,3$ satisfy (53). Still, purposefully, in order to allow a more direct comparison with common literature and with [30, we use the Gitman-Tyutin basis we costruct in the following. Furthermore, in this section, and only in this section, we choose $v^{\mu}=\gamma(c, 0,0, v)$. We choose $e_{\lambda}^{\mu}, \lambda=1,2$ as in the case of the polarization field. The third polarization can be chosen e.g. as follows (cf. [30]) in the case of the electromagnetic field:

$e_{3}^{\mu}=-i \frac{1}{|\mathbf{p}|} p^{\mu}, \quad p^{0}=|\mathbf{p}|$.

It is also useful to choose

$e_{0}^{\mu}=-i \frac{1}{2|\mathbf{p}|}(|\mathbf{p}|,-\mathbf{p})$.

Our choices are the same as in 30, apart from a slightly different normalization of both the vectors. We shall indicate the basis

$\left\{-i \frac{1}{2|\mathbf{p}|}(|\mathbf{p}|,-\mathbf{p}), e_{1}^{\mu}, e_{2}^{\mu},-i \frac{1}{|\mathbf{p}|} p^{\mu}\right\}$

as the Gitman-Tyutin basis. We stress that in this basis

$e_{0}^{\mu} e_{0}^{\nu} \eta_{\mu \nu}=0=e_{3}^{\mu} e_{3}^{\nu} \eta_{\mu \nu}$,

and

$e_{0}^{\mu} e_{3}^{\nu} \eta_{\mu \nu}=-1$.
We also get, in the case of the auxiliary field $B$,

$B=\int d^{4} p \frac{1}{S_{p}} \delta(D R)\left[\beta(\mathbf{p}) e^{i p x}+\right.$ h.c. $]$,

where $S_{p}$ is a suitable normalization. We remark that both $A^{\mu}$ and $B$ are considered as free fields, and then $\mathrm{DR}$ is a free field dispersion relation. Furthermore, the equation of motion for $B(15)$ implies that we can reduce to four the overall degrees of freedom for the fields $A^{\mu}, B$, and then only four operators in the set $a_{\lambda}, \beta$, with $\lambda=0,1,2,3$ are independent. We choose, in analogy with the discussion in [30] (sec. 4.2 therein), $a_{0}(\mathbf{p})=\beta(\mathbf{p})$.

Commutation relations are the same as in [30], as we are using the same basis for the electromagnetic sector of our model. We find

$\left[\beta(\mathbf{p}), \beta^{\dagger}(\mathbf{q})\right]=0$,

and

$\left[a_{3}(\mathbf{p}), \beta^{\dagger}(\mathbf{q})\right]=-\delta^{(3)}(\mathbf{p}-\mathbf{q})$,

and also

$\left[a_{\lambda}(\mathbf{p}), \beta^{\dagger}(\mathbf{q})\right]=0, \quad \lambda=1,2$.

Also, we find the following commutation brackets for $a_{\lambda}(\mathbf{p}), \lambda=1,2,3$ :

$\left[a_{\lambda}(\mathbf{p}), a_{\lambda^{\prime}}^{\dagger}(\mathbf{q})\right]=\delta_{\lambda \lambda^{\prime}} \delta^{(3)}(\mathbf{p}-\mathbf{q})$.

All other commutation relations are equal to zero. In particular, it holds

$\left[a_{3}(\mathbf{p}), a_{3}^{\dagger}(\mathbf{q})\right]=0$,

consistently with 30. We point out that in the Gitman-Tyutin basis, and in the Feynman gauge, one may recover the above commutation relations by first recovering the functional relations between the creation-distruction operators and the fields and their conjugate momenta. Cf. eg. chapter 7 in [42, in particular subsection 7.3.1 therein. We also recall that, a different choice of the basis with respect to the GitmanTyutin one, leads to different operators $\tilde{a}_{\lambda}, \tilde{a}_{\lambda}^{\dagger}$, which are linearly related (by means of a unitary transformation) to the ones of the Gitman-Tyutin basis. Moreover, the same commutation relations as above can be found also in a gauge with $\xi \neq 4 \pi[30$.

It is important to consider the particular form of the interaction term between the polarization field and the electromagnetic field. This term is proportional to $E^{\mu} P_{\mu}$.

We have to take into account that $P^{\mu}$ is constrained by the transversality condition with respect to the velocity $v^{\mu}$ (2). We can also introduce the projection operator

$\mathcal{P}^{\mu \nu}:=\eta^{\mu \nu}-\frac{v^{\mu} v^{\nu}}{v^{\rho} v_{\rho}}$, 
which is such that $v_{\mu} \mathcal{P}^{\mu \nu}=0$. It is evident that $\mathcal{P}^{\mu \nu} P_{\nu}=P^{\mu}$ and $\mathcal{P}^{\mu \nu} E_{\nu}=E^{\mu}$, so that the interaction term is transverse with respect to $v^{\mu}$. Furthermore, we are considering isotropic dielectric media, where $\mathbf{P} \propto \mathbf{E}$. Then, we expect also that $P^{\mu} \propto E^{\mu}$. Moreover, from the equations of motion we have

$\partial_{\mu} D^{\mu}=\partial_{\mu}\left(E^{\mu}+4 \pi \frac{g}{c} P^{\mu}\right)=0$.

This equation, in the homogeneous case (which represents the asymptotic limit in the comoving frame when a traveling perturbation is present), implies both $\partial_{\mu} E^{\mu}=0$ and $\partial_{\mu} P^{\mu}=0$, due to the aforementioned constitutive equation. As a consequence, by passing to the Fourier representation, this amounts to

$p_{\mu} E^{\mu}=0$,

i.e. also a transversality condition with respect to $p^{\mu}$ is implemented. If we introduce the further projection operator

$\overline{\mathcal{P}}^{\mu \nu}:=\eta^{\mu \nu}-\frac{p^{\mu} p^{\nu}}{p^{\rho} p_{\rho}}$,

we have also

$E^{\mu}=\overline{\mathcal{P}}^{\mu \nu} E_{\nu}$.

As a consequence, in the interaction term we have

$E^{\mu} P^{\nu} \eta_{\mu \nu}=\overline{\mathcal{P}}^{\mu \rho} E_{\rho} \mathcal{P}^{\nu \sigma} P_{\sigma} \eta_{\mu \nu}$

which means that the interaction term involves only polarizations which are transverse to both $v^{\mu}$ and $k^{\mu}$. In other terms, only physical transverse polarizations of the polarization field and of the electromagnetic field interact. Note that the projection operators $\mathcal{P}^{\mu \nu}, \overline{\mathcal{P}}^{\mu \nu}$ commute. The scalar polarization $\lambda=$ 0 , which involves only the electromagnetic field, and the longitudinal one $\lambda=3$, which involves both the electromagnetic field and the polarization field, correspond to interaction-free parts of the field, which decouple from the physical spectrum, as shown below. In particular, we stress that the longitudinal component of the polarization field does not participate to any physical process (it corresponds to a free oscillator which has no interaction with the electromagnetic field and that cannot enter any asymptotic (physical) scattering state).

We follow the discussion in [30] in order to construct states. As remarked above, in our framework, we have very important simplifications to be taken into account, due to the fact that our model is free (in terms of path integral approach, it is Gaussian), so that we can avoid discussing Ward identities, and also the infield formalism.

The space of states $\mathcal{R}$ is such that, given the vacuum state

$\beta(\mathbf{p})|0\rangle=a_{\lambda}(\mathbf{p})|0\rangle=b_{\lambda}(\mathbf{p})|0\rangle=0, \quad \lambda=1,2,3,(80)$ all states are spanned by states of the form

$\left(\beta^{\dagger}\right)^{m}\left(a_{\lambda}^{\dagger}\right)^{n}\left(b_{\lambda}^{\dagger}\right)^{l}|0\rangle, \quad \lambda=1,2,3$,

where for simplicity of notation we have left implicit the dependence on momenta of the operators and where $l, m, n \in \mathbb{N}$. The space $\mathcal{R}$ contains states with zero norm and negative norm as well, due to the fact that $a_{3}, a_{3}^{\dagger}, \beta, \beta^{\dagger}$ satisfy non-canonical commutation relations. We can easily confirm the presence of negative norm states as follows. Let us define

$d_{0}:=\frac{1}{\sqrt{2}}\left(a_{3}+\beta\right)$,

$d_{3}:=\frac{1}{\sqrt{2}}\left(a_{3}-\beta\right)$.

Then we obtain the commutation relations

$\left[d_{0}(\mathbf{p}), d_{0}^{\dagger}(\mathbf{q})\right]=-\delta^{(3)}(\mathbf{p}-\mathbf{q})$,

and also

$\left[d_{3}(\mathbf{p}), d_{3}^{\dagger}(\mathbf{q})\right]=\delta^{(3)}(\mathbf{p}-\mathbf{q})$.

The state $d_{0}^{\dagger}|0\rangle$ has negative norm:

$\left\langle 0\left|d_{0}(\mathbf{p}) d_{0}^{\dagger}(\mathbf{p})\right| 0\right\rangle=-\delta^{(3)}(0)$.

The space $\mathcal{R}$ is a space with indefinite metric. As remarked in [30, this is the price to be paid in order to get an explicit Lorentz covariance. One could consider only the subspace $\mathcal{R}_{\perp}$ of vectors of the form

$\left(a_{\lambda}^{\dagger}\right)^{n}\left(b_{\lambda^{\prime}}^{\dagger}\right)^{l}|0\rangle, \quad \lambda=1,2, \quad \lambda^{\prime}=1,2$,

which has a positive definite metric (it is an Hilbert space), but explicit covariance is lost. We also indicate the inverse formulas

$\beta=\frac{1}{\sqrt{2}}\left(d_{3}-d_{0}\right)$

$a_{3}=\frac{1}{\sqrt{2}}\left(d_{3}+d_{0}\right)$.

It is useful to define the physical space $\mathcal{R}_{p h}$, which is spanned by vectors of the form

$\left(\beta^{\dagger}\right)^{m}\left(a_{\lambda}^{\dagger}\right)^{n}\left(b_{\lambda^{\prime}}^{\dagger}\right)^{l}|0\rangle, \quad \lambda=1,2, \quad \lambda^{\prime}=1,2$.

It is a proper subspace of $\mathcal{R}$, because states generated by $a_{3}^{\dagger}$ and by $b_{3}^{\dagger}$ are absent. If we call $\mathcal{R}_{0}$ the subspace spanned by vectors (90) with $m \neq 0$, we have

$\mathcal{R}_{p h}=\mathcal{R}_{\perp} \oplus \mathcal{R}_{0}$.

States in $\mathcal{R}_{0}$ have zero norm and are orthogonal to any state in $\mathcal{R}_{p h}$. States in $\mathcal{R}_{p h}$ can be identified also by means of the condition

$\beta|\Psi\rangle=0, \quad|\Psi\rangle \in \mathcal{R}_{p h}$,

as $\beta$ commutes with any other operator except for $a_{3}^{\dagger}$. This condition amounts to the usual Gupta-Bleuler condition, as it can be expressed as follow:

$\hat{B}^{(+)}(t, \mathbf{x})|\Psi\rangle=0$, 
where $\hat{B}^{(+)}(t, \mathbf{x})$ is the positive frequency part of the operator $\hat{B}(t, \mathbf{x})$ (see 30] for details). The space $\mathcal{R}_{p h}$ can be described, as in [30, as a space whose non-zero norm vectors represent physical spaces, and vectors which differ for a zero-norm vector are physically equivalent:

$|\Phi\rangle \simeq|\Psi\rangle \Longleftrightarrow(|\Phi\rangle-|\Psi\rangle) \in \mathcal{R}_{0}$.

We take into account the construction of physical operators. In [30] it is shown that any physical operator $\hat{F}_{p h}$, i.e. any operator which is associated with a physical observable, should be such that

$\hat{F}_{p h} \mathcal{R}_{p h} \subset \mathcal{R}_{p h}$,

i.e. $\mathcal{R}_{p h}$ should be invariant. In particular, the Hamiltonian $\hat{H}$ should be a physical operator, and indeed it is. Let us suppose that $|\Psi\rangle \in \mathcal{R}_{p h}$. Then we have

$$
\begin{aligned}
\hat{B}^{(+)}(t, \mathbf{x}) \hat{H}|\Psi\rangle & =\left[\hat{B}^{(+)}(t, \mathbf{x}), \hat{H}\right]|\Psi\rangle \\
& =i \hbar \partial_{0} \hat{B}^{(+)}(t, \mathbf{x})|\Psi\rangle=0 .
\end{aligned}
$$

This ensures that the space $\mathcal{R}_{p h}$ is left invariant under the action of the Hamiltonian $\hat{H}$.

We point out also that, for any observable $\hat{F}$ which does not depend explicitly on time, in the Heisenberg picture it holds

$i \hbar \frac{\partial \hat{F}}{\partial t}=[\hat{F}, \hat{H}]$.

\subsection{The Hamiltonian and the Interaction Representation}

In the previous subsection, we have several times mentioned that we consider the fields as free. Implicitly, we have referred to the interaction representation, which is standard in quantum field theory as far as nontrivial interaction terms appear. There is no strict need to refer to this representation in our case, as the theory can be dealt with exactly. Still, there are nontrivial subtleties, which are related to the choice of the gauge. As is known, in standard QED the usual gauge for quantum electrodynamics is the so called Feynman gauge, i.e. $\xi=4 \pi$. This gauge avoids to be faced with the appearance of the so-called ghost pole in the theory [41, which represents a nontrivial and quite hard problem to be dealt with. In the present case, the choice $\xi=4 \pi$ is effective only in the interaction representation, in the sense that in the Heisenberg representation it is not available, as it is not difficult to realize. We defer the study in the latter representation to a future publication, as even in the homogeneous case (i.e. no traveling perturbation) there are many tricky formal problems which require extensive and long calculations. Herein, in the spirit of most books and studies in condensed matter physics, we adopt the interaction representation, so that field operators evolve freely, as dictated by the free Hamiltonian operator, and states evolve through the Dyson evolution operator which is constructed by means of the interaction term in the Hamiltonian. By referring to our case, we have

$H_{0}=H_{e m}+H_{p o l}$,

where $H_{0}$ is the free Hamiltonian contribution, with

$$
\begin{aligned}
H_{e m} & =\int d^{3} x\left[2 \pi c^{2}\left(\Pi_{A}^{i}\right)^{2}+\frac{1}{16 \pi} F_{i j} F^{i j}\right. \\
& \left.+c A_{0}\left(\partial_{i} \Pi_{A} i\right)-B\left(\partial_{i} A^{i}\right)-\frac{\xi}{2} B^{2}\right],
\end{aligned}
$$

and

$$
\begin{aligned}
H_{p o l} & =\int d^{3} x\left[-\frac{\chi \omega_{0}^{2} c^{2}}{2\left(v^{0}\right)^{2}} \Pi_{P \mu} \Pi_{P}^{\mu}-\frac{1}{2 \chi} P_{\mu} P^{\mu}\right. \\
& \left.-c \frac{v^{k}}{v_{0}}\left(\partial_{k} P^{\mu}\right) \Pi_{P \mu}+\frac{2 \pi g^{2}}{c^{2}}\left(M_{0 i}\right)^{2}\right],
\end{aligned}
$$

where we have defined

$M_{\alpha \beta}:=v_{\alpha} P_{\beta}-v_{\beta} P_{\alpha}$.

The interaction term is

$$
\begin{aligned}
H_{i n t} & =\int d^{3} x\left[\frac{g}{c} M_{0 i} F^{0 i}+\frac{g}{2 c} M_{i j} F^{i j}\right] \\
& =\int d^{3} x\left[\frac{g}{2 c} M_{\mu \nu} F^{\mu \nu}\right]=\int d^{3} x\left[\frac{g}{c} P_{\mu} E^{\mu}\right] .
\end{aligned}
$$

One has to take into account that, in the interaction representation, $\Pi_{A}^{i}$ is considered at $g=0$, so a simplification in calculations occurs. Note that in (100) the last term has been considered as a further contribution to the free Hamiltonian operator, despite the fact that it is proportional to $g^{2}$. This is due to the fact that this term 'renormalizes' the proper frequency $\omega_{0}$ of the polarization field and, substantially, is a sort of improvement of the standard free Hamiltonian. See e.g. [5, 7, 40,

Furthermore, the Hamiltonian $H_{e m}$ can be simplified by using the equations of motion for the electromagnetic field: indeed, the last three terms on the solutions of the equations of motion become

$$
\begin{gathered}
c A_{0}\left(\partial_{i} \Pi_{A i}\right)-B\left(\partial_{i} A^{i}\right)-\frac{\xi}{2} B^{2} \\
=B \partial_{0} A_{0}-A_{0} \partial_{0} B+\frac{\xi^{2}}{2} B^{2}
\end{gathered}
$$

and this is true both in the interaction representation with $g=0$ and in full interacting case, as a direct inspection confirms. Then we obtain

$$
\begin{aligned}
H_{e m} & =\int d^{3} x\left[2 \pi c^{2}\left(\Pi_{A}^{i}\right)^{2}+\frac{1}{16 \pi} F_{i j} F^{i j}\right. \\
& \left.+B \partial_{0} A_{0}-A_{0} \partial_{0} B+\frac{\xi^{2}}{2} B^{2}\right],
\end{aligned}
$$

where one can immediately realize that the last three terms in the free electromagnetic Hamiltonian do contribute only to the unphysical polarizations, and then are substantially irrelevant for the physics at hand 
(their role is only formal, being a consequence of the covariance requirement, as seen).

We display the explicit expression of the Hamiltonian in terms of creation and annihilation operators. We recall our choices for the bases: the GitmanTyutin basis (63) for the electromagnetic field, and $\left\{e_{1}^{\mu}, e_{2}^{\mu}, \frac{v^{\mu}}{c}-p^{\mu} \frac{c}{\omega}\right\}$ for the polarization field. We first fix the normalizations for the fields:

$P^{\mu}=\sum_{\lambda=1}^{3} \int \frac{d^{3} p}{(2 \pi)^{3 / 2}} \sqrt{\frac{\chi \omega_{0}^{2}}{2 \Omega_{0}}}\left[e_{\lambda}^{\mu} b_{\lambda}(\mathbf{p}) e^{-i p x}+\right.$ h.c. $]$,

where the renormalized frequency is

$\Omega=\omega_{0} \sqrt{1+4 \pi g^{2} \chi}$

and, for the electromagnetic field and the auxiliary field we get

$A^{\mu}=\sum_{\lambda=0}^{3} \int \frac{d^{3} p}{(2 \pi)^{3 / 2}} \sqrt{\frac{(2 \pi)}{p_{0}}}\left[e_{\lambda}^{\mu} a_{\lambda}(\mathbf{p}) e^{-i p x}+h . c.\right]$

$B=\frac{1}{4 \pi} \int \frac{d^{3} p}{(2 \pi)^{3 / 2}} \sqrt{\frac{(2 \pi)}{p_{0}}} p_{0}\left[\beta(\mathbf{p}) e^{-i p x}+\right.$ h.c. $]$.

In the following, each contribution to the Hamiltonian is distinguished between transversal and nontrasversal. We know that $\hat{H}$ is at most quadratic in the creation and annihilation operators which span the space $\mathcal{R}$. Moreover, it can be written as follows:

$\hat{H}=\hat{H}_{p h}+\hat{H}^{\prime}$,

where $\hat{H}_{p h}$ corresponds to the physical part of the Hamiltonian, which is the same as in the Coulomb gauge (cf. 30 for QED), and involves only transverse polarizations. $\hat{H}^{\prime}$ is the remaining part, which involves unphysical polarizations, which are decoupled from the physical spectrum.

We develop our calculations for the specific case $v^{\mu}=$ $(c, 0,0,0)$ for simplicity; we get:

$\hat{H}_{e m, p h}=\int d^{3} p p_{0} \sum_{\lambda=1}^{2} a_{\lambda}^{\dagger}(\mathbf{p}, t) a_{\lambda}(\mathbf{p}, t)$,

$\hat{H}_{p o l, p h}=\int d^{3} p \Omega_{0} \sum_{\lambda=1}^{2} b_{\lambda}^{\dagger}(\mathbf{p}, t) b_{\lambda}(\mathbf{p}, t)$,

for the free part, and

$$
\begin{aligned}
\hat{H}_{i n t, p h}= & i \frac{g}{c} \int d^{3} p \sqrt{\frac{\pi \chi \omega_{0}^{2}}{p_{0} \Omega}} c p_{0} \sum_{\lambda=1}^{2} \\
& {\left[\left(a_{\lambda}(\mathbf{p}, t) b_{\lambda}(-\mathbf{p}, t)-a_{\lambda}^{\dagger}(\mathbf{p}, t) b_{\lambda}^{\dagger}(-\mathbf{p}, t)\right)+\right.} \\
& \left.\left(a_{\lambda}(\mathbf{p}, t) b_{\lambda}^{\dagger}(\mathbf{p}, t)-a_{\lambda}^{\dagger}(\mathbf{p}, t) b_{\lambda}(\mathbf{p}, t)\right)\right] \cdot(111)
\end{aligned}
$$

for the interaction term. We recall that the operators $a_{\lambda}(\mathbf{p}, t)$ in the interaction representation are related to the ones $a_{\lambda}(\mathbf{p})$ in the Schroedinger and the ones $a_{\lambda}^{h}(\mathbf{p}, t)$ in the Heisenberg representations as follows:

$a_{\lambda}(\mathbf{p}, t)=e^{i \hat{H}_{0} t} a_{\lambda}(\mathbf{p}) e^{-i \hat{H}_{0} t}$,

$a_{\lambda}^{h}(\mathbf{p}, t)=e^{i \hat{H} t} a_{\lambda}(\mathbf{p}) e^{-i \hat{H} t}$.

Analogous formulas hold for $\beta(\mathbf{p}, t), a_{3}(\mathbf{p}, t), b_{\lambda}(\mathbf{p}, t)$. The contributions to the non-transversal (unphysical) part of the Hamiltonian are (we omit to use explicit arguments when no ambiguity occurs)

$\hat{H}_{e m}^{\prime}=\int d^{3} p\left[\left(-\frac{p_{0}}{4}\right) \beta^{\dagger} \beta\right]$

for the electromagnetic field, and

$\hat{H}_{\text {pol }}^{\prime}=\int d^{3} p \Omega_{0} b_{3}^{\dagger} b_{3}$

for the polarization part. For the interaction term we get

$$
\begin{aligned}
& \hat{H}_{\text {int }}^{\prime}=\frac{g}{c} \int d^{3} p \sqrt{\frac{\pi \chi \omega_{0}^{2}}{p_{0} \Omega}} \\
& \left(\frac { 1 } { 2 } c p _ { 0 } \left[\left(\beta(\mathbf{p}, t) b_{3}(-\mathbf{p}, t)+\beta^{\dagger}(\mathbf{p}, t) b_{3}^{\dagger}(-\mathbf{p}, t)\right)+\right.\right. \\
& \left.\left.\left(\beta(\mathbf{p}, t) b_{3}^{\dagger}(\mathbf{p}, t)+\beta^{\dagger}(\mathbf{p}, t) b_{3}(\mathbf{p}, t)\right)\right]\right) .
\end{aligned}
$$

It is important to underline that matrix elements between physical states of the $H^{\prime}$ operators vanish:

$\left\langle\Phi\left|H^{\prime}\right| \Psi\right\rangle=0, \quad|\Phi\rangle,|\Psi\rangle \in \mathcal{R}_{p h}$.

Indeed, the term in $b_{3}^{\dagger} b_{3}$ vanishes because physical states don't contain any longitudinal quanta of polarization by construction. The other terms vanish as well. It may be interesting to point out that, in our construction, the condition

$\left(\partial_{\mu} P^{\mu}\right)^{(+)}|\Psi\rangle=0$

is implemented for any $|\Psi\rangle \in \mathcal{R}_{p h}$. This condition can be understood as a transversality condition in a weak sense (as only for $\lambda=1,2$ the polarization field implements the transversality condition automatically, with $p_{\mu} e_{\lambda}^{\mu}=0$. Cf. the discussion which follows eq. (75) ).

As to the physical part, we can find a linear canonical transformation (a Bogoliubov transformation, known as Fano transformation for this specific model [5, 6]), which carries $\hat{H}_{p h}$ into a simplified form involving quasi-particle states $\alpha_{\lambda}(\mathbf{p})$, with $\lambda=1,2$. In particular, as in the original Hopfield paper [5], we impose for $\lambda=1,2$

$\left[\alpha_{\lambda}(\mathbf{p}), \hat{H}_{p h}\right]=\omega(\mathbf{p}) \alpha_{\lambda}(\mathbf{p})$,

and the corresponding commutation relations are

$\left[\alpha_{i}(\mathbf{p}), \alpha_{j}^{\dagger}(\mathbf{q})\right]=\delta_{i j} \delta^{(3)}(\mathbf{p}-\mathbf{q})$.

In this way we can recover the same dispersion relation as for the full model, as it is easy to verify. Cf. also [40. 


\subsection{A more general setting}

In concluding this section, we point out that the above model can be easily extended to the case of $N>1$ material harmonic oscillators coupled with the electromagnetic field. The simple substitutions $P^{\mu} \mapsto$ $P_{(k)}^{\mu}$, with $k=1, \ldots, N, \omega_{0} \mapsto \omega_{0(k)}, \chi \mapsto \chi_{(k)}$ and $g \mapsto g_{(k)}$, lead to the desired form of the Lagrangian:

$$
\begin{aligned}
\mathcal{L}_{c}: & =-\frac{1}{16 \pi} F_{\mu \nu} F^{\mu \nu} \\
& -\sum_{k=1}^{N}\left[\frac{1}{2 \chi_{(k)} \omega_{0(k)}^{2}}\left[\left(v^{\rho} \partial_{\rho} P_{(k) \mu}\right)\left(v^{\sigma} \partial_{\sigma} P_{(k)}^{\mu}\right)\right]\right. \\
& \left.-\frac{1}{2 \chi_{(k)}} P_{(k) \mu} P_{(k)}^{\mu}+\frac{g_{(k)}}{2 c}\left(v_{\mu} P_{(k) \nu}-v_{\nu} P_{(k) \mu}\right) F^{\mu \nu}\right] \\
& +B\left(\partial_{\mu} A^{\mu}\right)+\frac{\xi}{2} B^{2}+\sum_{k=1}^{N} \lambda_{(k)}\left(v_{\mu} P_{(k)}^{\mu}\right) .
\end{aligned}
$$

At the level of the constraints, in place of $\Gamma_{3}, \Gamma_{4}$ we get $2 \mathrm{~N}$ constraints $\Gamma_{3(k)}, \Gamma_{4(k)}$, and, analogously, $2 \mathrm{~N}$ constraints $\Gamma_{5(k)}, \Gamma_{6(k)}$. Fields $P_{(k)}^{\mu}$ satisfy:

$$
\begin{aligned}
& \left\{P_{(k)}^{\mu}, \Pi_{P_{(l)}}^{\nu}\right\}_{D} \\
& :=\delta_{(k)(l)}\left(\eta^{\mu \nu}-\frac{1}{v_{\rho} v^{\rho}} v^{\mu} v^{\nu}\right) \delta^{(3)}(\mathbf{x}-\mathbf{y}) .
\end{aligned}
$$

\section{Example: model with a $v=$ const traveling dielectric perturbation}

Let us consider the case where the dielectric perturbation induced by means of the Kerr effect is traveling with constant velocity $v$ in the lab frame. We can also assume that dependence on transverse coordinates $y, z$ is absent (which means that our dielectric perturbation is actually modelized as a dielectric slab infinitely extended in transverse directions). The relevance of a covariant approach is easily appreciated by taking into account that, in the reference frame comoving with the perturbation, one gets a static dependence of the parameters $\chi, \omega_{0}, g$ on $x^{\prime} / \gamma$, where $x^{\prime}=\gamma(x-v t), t^{\prime}=\gamma\left(t-\frac{v}{c^{2}} x\right)$ represent the Lorentz boost connecting lab and comoving frame. As a consequence of this, it is easily understood that energy is conserved in the comoving frame, i.e. it is possible to perform a variable separation involving the time coordinate in such a way that the energy $\omega^{\prime}$ in the comoving frame is conserved. This result is very important and helpful in interpreting scattering in presence of the perturbation, and amply corroborates the relevance of a covariant approach and a consistent quantization. In particular, it is possible to quantize the system and to find out a scattering basis for the quantum fields in a straightforward way, without any problem arising because of a possible time-dependence of the perturbation. Moreover, covariance, together with a correct quantization, allows to find out a conserved inner product whose associated norm is fundamental in defining particles and antiparticles for the given model. Note that the norm sign is independent on the frame chosen, and then is an invariant concept (at it should be).

A further paper is dedicated to results and analysis about this specific model, with special reference to the question of analogous Hawking radiation in dielectric media 23]. We limit ourselves to point out that, as far as the full model with the uniformly travelling perturbation is concerned, in the lab frame one can still implement quantization by means of standard canonical commutation relations. Indeed, the electromagnetic part can be quantized in the Coulomb gauge, without any change with respect to the standard strategy (se e.g. [5], or even [9, 10] for a more involved model). The polarization field part does not require a particular care in the definition of the variable conjugate to $\mathbf{P}$, which is obtained by standard tools of Lagrangian formalism. One is lead to the following equal time commutation relations:

$\left[P_{i}(t, \mathbf{x}), \frac{1}{\chi \omega_{0}^{2}(t, \mathbf{y})} \partial_{t} P_{j}(t, \mathbf{y})\right]=i \hbar \delta_{i j} \delta^{(3)}(\mathbf{x}-\mathbf{y})$.

It can be easily shown that the conjugate momentum $\frac{1}{\chi \omega_{0}^{2}} \partial_{t} P_{i}$ leads to correct Hamiltonian equations for the polarization field.

We stress again that, in the lab, the presence of the travelling perturbation induces an explicit dependence on $t$ of the total Hamiltonian of the model. This implies that energy is not conserved in this frame, still one can expect that some sort of conservation occurs (cf. e.g. what happens in the case of the generalized ManleyRowe identities [23]). This makes the quantization in the lab less clear and more problematic than the one in the comoving frame.

\section{Asymptotic behavior of solutions in the comoving frame for $v=$ const}

The Hamiltonian (21) allows variables separation for the solutions. Defining:

$A^{\mu}(x, y, z, t)=e^{-i \omega t+i k_{y} y+i k_{z} z} a^{\mu}(x)$,
$P^{\mu}(x, y, z, t)=e^{-i \omega t+i k_{y} y+i k_{z} z} p^{\mu}(x)$,
$B(x, y, z, t)=e^{-i \omega t+i k_{y} y+i k_{z} z} b(x)$,
$\lambda(x, y, z, t)=e^{-i \omega t+i k_{y} y+i k_{z} z} l(x)$

we obtain a second order system of ordinary differential equations for the variables $a^{\mu}(x), p^{\mu}(x), b(x)$ and $l(x)$. Since the equation for $l(x)$ is $l(x)=0$ we can omit this variable from the system. The equation involving $b(x)$ is algebraic and can be used to simplify the other eight. To this second order system we can associate a first order one by introducing:

$\alpha^{\mu}(x):=\partial_{x} a^{\mu}(x), \quad \pi^{\mu}(x):=\partial_{x} p^{\mu}(x)$,

$\beta(x):=\partial_{x} b(x)$, 
then if $W(x):=\left(a^{\mu}(x), \alpha^{\mu}(x), p^{\mu}(x), \pi^{\mu}(x)\right)$, we obtain the following system:

$W^{\prime}(x)=K_{16} W(x)$,

where $K_{16}$ is a suitable $16 \times 16$ operator. This can be written as $K_{16}=\mathcal{C}+\mathcal{R}(x)$, where $\mathcal{C}$ is a constant $16 \times 16$ matrix and $\mathcal{R}(x)$ contains the non constant part. For simplicity, let us consider the case where only dielectric susceptibility varies; then $\mathcal{R}(x)$ has the form:

$\mathcal{R}(x):=\left(\begin{array}{cccc}0_{4} & 0_{4} & 0_{4} & 0_{4} \\ A_{4} & B_{4} & C_{4} & D_{4} \\ 0_{4} & 0_{4} & 0_{4} & 0_{4} \\ 0_{4} & 0_{4} & 0_{4} & 0_{4}\end{array}\right)$,

where $0_{4}$ is the $4 \times 4$ identity matrix, and $A_{4}=$ $-\frac{i v^{0} \omega \omega_{0}^{2} \chi(x)}{c\left(v^{1}\right)^{2}} I_{4}, B_{4}=\frac{\omega_{0}^{2} \chi(x)}{c v^{1}} I_{4}, C_{4}=-\frac{i v^{0} \omega \chi^{\prime}(x)}{v^{1} \chi(x)} I_{4}$, $D_{4}=\frac{\chi^{\prime}(x)}{\chi(x)} I_{4}$, with $I_{4}$ the $4 \times 4$ identity matrix. Under the hypothesis:

$\int_{a}^{\infty} d x|\mathcal{R}(x)|<\infty$

which physically can match very well the nature of travelling perturbation of $\delta \chi$ (see e.g. the theory displayed in [43]), we can infer that, both as $x \rightarrow$ $\infty$ and as $x \rightarrow-\infty$, the asymptotic behavior of solutions is governed by the eigenvalues of $\mathcal{C}$, which implies that the basis for $\delta \chi=0$ is asymptotically a good scattering basis also for the perturbed problem. To be more precise: the asymptotic region solutions are a scattering basis, and, moreover, solutions of the full equations asymptotically behave as the asymptotic region solutions, which then represent a good scattering basis.

\section{Conclusions}

We have presented a model aimed to a semiphenomenological description of quantum electrodynamics in presence of a dielectric perturbation in a dielectric medium. The standard Hopfield model has been made fully covariant, and its quantization procedure has been discussed in detail. We stress that the requirement of covariance is fundamental in order to allow a proper interpretation of measurable quantities (e.g. quantum probabilities) as viewed by different (inertial) observers. E.g., in the discussion of the analogue Hawking effect in dielectrics, a very important conceptual tool consists in the analysis one performs in the comoving frame of the dielectric perturbation induced by the Kerr effect. This request for covariance reflects itself in a more tricky quantization procedure, which involves both the electromagnetic field, which represents a constrained system, as any gauge theory, and, as such, requires a special quantization procedure, and also the polarization field, due to a further constraint it carries into the lagrangian of the system. We have dealt the problem both in a non-covariant approach and in a covariant one. We have also discussed how to identify the asymptotic behaviour of the solutions.

Our direct developments of the present work include: 1) a perturbative approach [45. for the model, 2) an application of the aforementioned approach to the problem of photon pair creation by a helicoidal rotating dielectric perturbation [46, 3) the nonperturbative study of the analogue Hawking effect [23], together with the elaboration of a simplified model reproducing the basic features of the full Hopfield model discussed here. 4) The description, at the perturbative level, of cosmological analogue situations [47.

\section{References}

[1] W. Heisenberg and H. Euler, Zeitschr. Phys. 98, 714 (1936). English translation in arXiv:physics/0605038

[2] J. Schwinger, Phys. Rev. 82, 664 (1951).

[3] J. Schwinger, Proc. Natl. Acad. Sci. 89, 40914093 (1992); 89, 1111811120 (1992); 90, 958959 (1993); 90, 21052106 (1993); 90, 45054507 (1993); 90, 72857287 (1993); 91, 64736475 (1994).

[4] A. Luks, V. Perinová, Quantum Aspects of Light Propagation. Springer, Berlin (2009).

[5] J.J.Hopfield, Phys. Rev. 112, 1555 (1958).

[6] U.Fano, Phys. Rev. 103, 1202 (1956).

[7] C.Kittel, Quantum theory of solids. Wiley, New York (1987).

[8] A.S.Davydov, Teoria del solido. Mir, Moscow (1984).

[9] B.Huttner and S.M.Barnett, Phys. Rev. A 46, 4306 (1992).

[10] L.G. Suttorp and A.J. van Wonderen, Europhys. Lett. 67, 766 (2004).

[11] L.G. Suttorp, J. Phys. A: Math. Theor. 40, 3697 (2007).

[12] R.W.Boyd, Nonlinear Optics. 3rd ed. Academic Press, New York (2008).

[13] T. G. Philbin, C. Kuklewicz, S. Robertson, S. Hill, F. Konig and U. Leonhardt, Science 319, 1367 (2008) arXiv:0711.4796 [gr-qc]].

[14] F. Belgiorno, S.L. Cacciatori, M.Clerici, V. Gorini, G. Ortenzi, L. Rizzi, E. Rubino, V.G. Sala, and D. Faccio, Phys. Rev. Lett. 105203901 (2010).

[15] E.Rubino, F.Belgiorno, S.L.Cacciatori, M.Clerici, V.Gorini, G.Ortenzi, L.Rizzi, V.G.Sala, M.Kolesik, J.V.Moloney, D.Faccio, New J. Phys. 13085005 (2011).

[16] F. Belgiorno, S. L. Cacciatori, G. Ortenzi, L. Rizzi, V. Gorini and D. Faccio, Phys. Rev. D 83, 024015 (2011) arXiv:1003.4150 [quant-ph]].

[17] M. Petev, N. Westerberg, D. Moss, E. Rubino, C. Rimoldi, S. L. Cacciatori, F. Belgiorno and D. Faccio, Phys. Rev. Lett. 111, 043902 (2013).

[18] S.Finazzi and I.Carusotto, Phys. Rev. A87, 023803 (2013).

[19] S. Finazzi and I. Carusotto, Phys. Rev. A 89, 053807 (2014) arXiv:1303.4990 [physics.optics]].

[20] H. Minkowski, Die Grundgleichungen für die elektromagnetischen Vorgänge in bewegten Körpern. Nachrichten von der Gesellschaft der Wissenschaften zu Göttingen, Mathematisch-Physikalische Klasse. S. 53111 (1908).

[21] E.J. Post, Formal structure of electromagnetics: general covariance and electromagnetics. North-Holland, Amsterdam (1962).

[22] P.Penfield and H.A.Haus, Electrodynamics of moving media. M.I.T. Press, Cambridge, Massachussetts (1967).

[23] F.Belgiorno, S.L.Cacciatori, F.Dalla Piazza, Phys. Rev. D 91, 124063 (2015).

[24] W.Gordon, Ann. Phys. (Leipzig), 72, 421456, (1923). 
[25] N.L.Balazs, Jour. Optical Soc. Amer. 45, 63 (1955).

[26] Pham Mau Quan, Archives for Rational Mechanics and Analysis 1, 54-80 (1957/58).

[27] J.L. Synge, Geometrical optics in moving dispersive media. Commun.Dublin Inst.Ser.A 12 (1956).

[28] C.Barceló, S.Liberati, M.Visser, Living Rev. Relativity 14, 3 (2011).

[29] S.A.R.Horsley, Phys. Rev. A 86, 023830 (2012)

[30] D.M.Gitman and I.V.Tyutin, Quantization of Fields with Constraints. Springer Series in Nuclear and Particle Physics, Springer, Berlin (1990).

[31] M.Henneaux and C.Teitelboim, Quantization of Gauge Systems. Princeton University Press, Princeton (1994).

[32] K. M. Watson and J. M. Jauch, Phys. Rev. 75, 1249 (1949).

[33] S.R.De Groot and L.G.Suttorp, Foundations of Electrodynamics. Elsevier (1972).

[34] C.Itzykson and J-C.Zuber, Quantum Field Theory. McGraw Hill (1980).

[35] P.A.M. Dirac, Lectures on Quantum Mechanics. Belfer Graduate School of Science, Yeshiva University. New York, 1964.

[36] P.A.M. Dirac, Can. J. Math. 2, 129 (1950); ibid. 3, 1 (1951).

[37] Heinz J. Rothe and Klaus D. Rothe, Classical and Quantum Dynamics of Constrained Hamiltonian Systems. World Scientific, Singapore (2010).

[38] J.Barcelos-Neto, Ashok Das and W.Scherer, Acta Phys. Pol. B18 269 (1987).

[39] J.Dimock, Quantum Mechanics and Quantum Field Theory. Cambridge University Press, Cambridge (2011).

[40] B.Huttner,J.J. Baumberg and S.M.Barnett, Europhys. Lett. 16, 177 (1991).

[41] N.Nakanishi and I. Ojima, Covariant Operator Formalism of Gauge Theories and Quantum Gravity. Wolrd Scientific, Singapore (1990).

[42] W.Greiner and J.Reinhardt, Field Quantization. Springer, Berlin (1996)

[43] M.S.F. Eastham, The Asymptotic Solution of Linear Differential Systems. Applocations of the Levinson Theorem. London Mathematical Society monographs. New series 4. Clarendon Press, Oxford (1989).

[44] C.A.Manogoue, Ann. Phys. 181, 261 (1988).

[45] F. Belgiorno, S. L. Cacciatori and F. Dalla Piazza, Eur. Phys. J. D 68, 134 (2014) arXiv:1402.2838 [quant-ph]].

[46] F.Dalla Piazza et al., forthcoming.

[47] N. Westerberg, S. Cacciatori, F. Belgiorno, F. Dalla Piazza and D. Faccio, New J. Phys. 16, 075003 (2014) arXiv:1403.5910 [gr-qc]]. 